Supporting Information

\title{
Parametrization of Nonbonded Force Field Terms for Metal- Organic Frameworks Using Machine Learning Approach
}

Vadim V. Korolev, ${ }^{\dagger}, *$ Yuriy M. Nevolin, ${ }^{\dagger}$ Thomas A. Manz, ${ }^{\perp}$ and Pavel V. Protsenko ${ }^{\dagger}$

${ }^{\dagger}$ Department of Chemistry, Lomonosov Moscow State University, Moscow 119991, Russia

Frumkin Institute of Physical Chemistry and Electrochemistry, Russian Academy of Sciences, Moscow 119071, Russia

${ }^{\perp}$ Department of Chemical \& Materials Engineering, New Mexico State University, Las Cruces, New Mexico 88003-8001, United States

*The author to whom correspondence should be addressed: korolev@ colloid.chem.msu.ru 

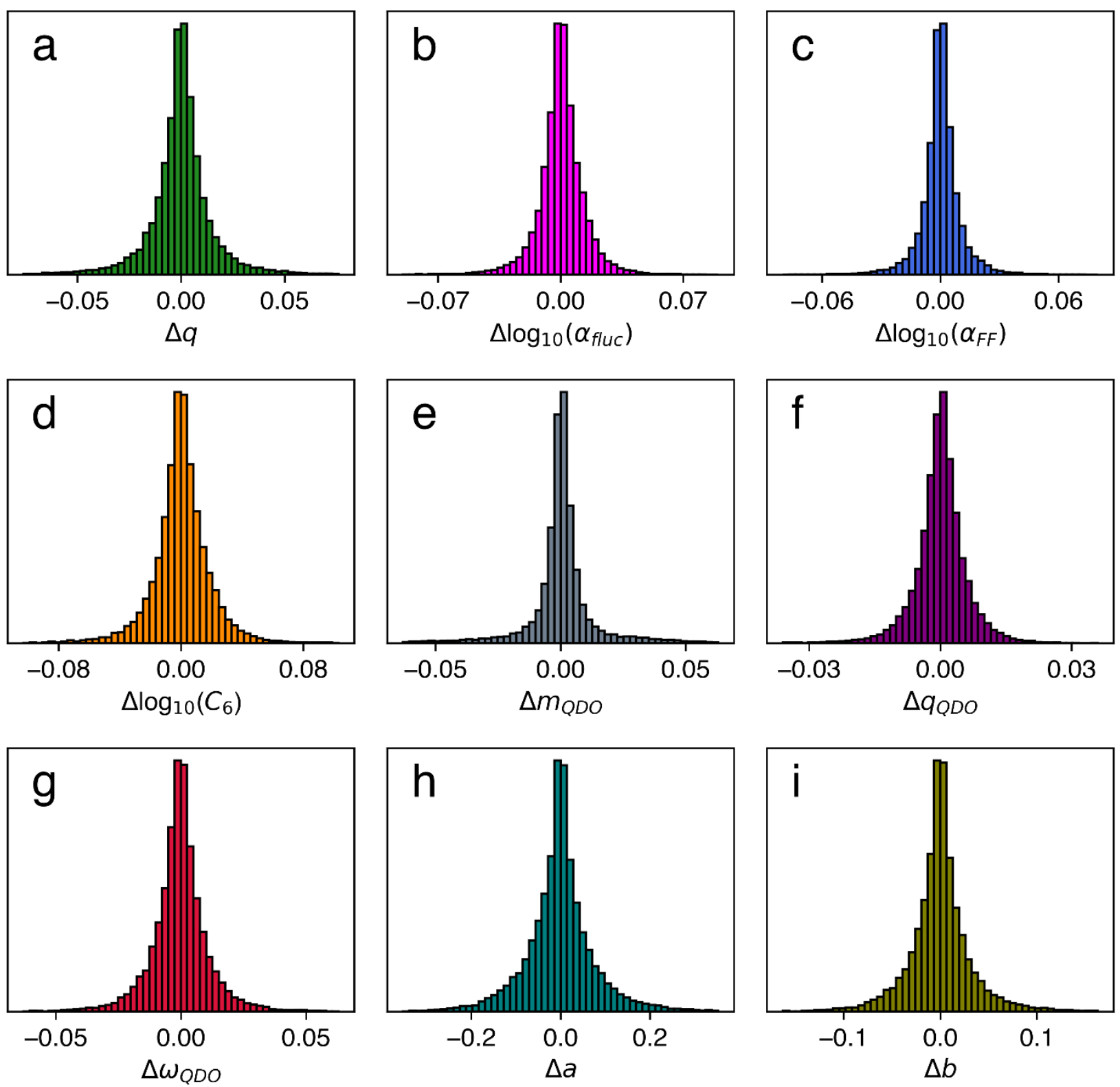

Figure S1. Histograms of the deviations of the predicted FF precursor values from the reference values: (a) partial charge, (b) fluctuating polarizability, (c) FF polarizability, (d) dispersion coefficient $C_{6}$, (e) QDO mass, (f) QDO charge, (g) QDO frequency, (h) electron cloud parameter $a$, and (i) electron cloud parameter $b$. 
Table S1. List of used fingerprints.

\begin{tabular}{|c|c|}
\hline \multirow{8}{*}{ 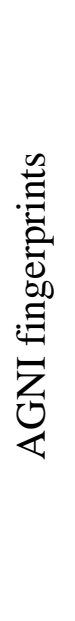 } & Gaussian function width $\eta=0.8$ \\
\hline & Gaussian function width $\eta=1.23$ \\
\hline & Gaussian function width $\eta=1.88$ \\
\hline & Gaussian function width $\eta=2.89$ \\
\hline & Gaussian function width $\eta=4.43$ \\
\hline & Gaussian function width $\eta=6.8$ \\
\hline & Gaussian function width $\eta=10.4$ \\
\hline & Gaussian function width $\eta=16.0$ \\
\hline & coordination likelihood $w_{\mathrm{CN}=1}$ \\
\hline & coordination likelihood $w_{\mathrm{CN}=2}$ \\
\hline & coordination likelihood $w_{\mathrm{CN}=3}$ \\
\hline & coordination likelihood $w_{\mathrm{CN}=4}$ \\
\hline & coordination likelihood $w_{\mathrm{CN}=5}$ \\
\hline & coordination likelihood $w_{\mathrm{CN}=6}$ \\
\hline & coordination likelihood $w_{\mathrm{CN}=7}$ \\
\hline & coordination likelihood $w_{\mathrm{CN}=8}$ \\
\hline & coordination likelihood $w_{\mathrm{CN}=9}$ \\
\hline & coordination likelihood $w_{\mathrm{CN}=10}$ \\
\hline & coordination likelihood $w_{\mathrm{CN}=11}$ \\
\hline & coordination likelihood $w_{\mathrm{CN}=12}$ \\
\hline & coordination likelihood $w_{\mathrm{CN}=13}$ \\
\hline & coordination likelihood $w_{\mathrm{CN}=14}$ \\
\hline & coordination likelihood $w_{\mathrm{CN}=15}$ \\
\hline & coordination likelihood $w_{\mathrm{CN}=16}$ \\
\hline$\stackrel{\Xi}{\Xi}$ & coordination likelihood $w_{\mathrm{CN}=17}$ \\
\hline$\stackrel{\infty}{0}$ & coordination likelihood $w_{\mathrm{CN}=18}$ \\
\hline 至 & coordination likelihood $w_{\mathrm{CN}=19}$ \\
\hline$\overline{\tilde{J}}$ & coordination likelihood $w_{\mathrm{CN}=20}$ \\
\hline$\dot{U}$ & coordination likelihood $w_{\mathrm{CN}=21}$ \\
\hline
\end{tabular}




\begin{tabular}{|c|c|}
\hline & coordination likelihood $w_{\mathrm{CN}=22}$ \\
\hline & coordination likelihood $w_{\mathrm{CN}=23}$ \\
\hline & coordination likelihood $w_{\mathrm{CN}=24}$ \\
\hline & first ionization energy of site \\
\hline & electronegativity of site \\
\hline & number of first neighbors \\
\hline & averaged first ionization energy of first neighbors \\
\hline $\overrightarrow{\bar{D}}$ & averaged electronegativity of first neighbors \\
\hline 江 & averaged distance to first neighbors \\
\hline & number of second neighbors \\
\hline & averaged first ionization energy of second neighbors \\
\hline & averaged electronegativity of second neighbors \\
\hline & averaged distance to second neighbors \\
\hline & single bond motif type, $\mathrm{CN}=1$ \\
\hline & L-shaped motif type, $\mathrm{CN}=2$ \\
\hline & water-like motif type, $\mathrm{CN}=2$ \\
\hline & bent $120^{\circ}$ motif type, $\mathrm{CN}=2$ \\
\hline & bent $150^{\circ}$ motif type, $\mathrm{CN}=2$ \\
\hline & linear motif type, $\mathrm{CN}=2$ \\
\hline & trigonal planar motif type, $\mathrm{CN}=3$ \\
\hline & trigonal non-coplanar motif type, $\mathrm{CN}=3$ \\
\hline & T-shaped motif type, $\mathrm{CN}=3$ \\
\hline & square co-planar motif type, $\mathrm{CN}=4$ \\
\hline & tetrahedral motif type, $\mathrm{CN}=4$ \\
\hline & rectangular see-saw-like motif type, $\mathrm{CN}=4$ \\
\hline & see-saw-like motif type, $\mathrm{CN}=4$ \\
\hline$\Xi$ & trigonal pyramidal motif type, $\mathrm{CN}=4$ \\
\hline$\stackrel{\infty}{=}$ & pentagonal planar motif type, $\mathrm{CN}=5$ \\
\hline$\stackrel{\oplus}{=}$ & square pyramidal motif type, $\mathrm{CN}=5$ \\
\hline$\overline{0}$ & trigonal bipyramidal motif type, $\mathrm{CN}=5$ \\
\hline
\end{tabular}




\begin{tabular}{|c|c|}
\hline & hexagonal planar motif type, $\mathrm{CN}=6$ \\
\hline & octahedral motif type, $\mathrm{CN}=6$ \\
\hline & pentagonal pyramidal motif type, $\mathrm{CN}=6$ \\
\hline & hexagonal pyramidal motif type, $\mathrm{CN}=7$ \\
\hline & pentagonal bipyramidal motif type, $\mathrm{CN}=7$ \\
\hline & body-centered cubic motif type, $\mathrm{CN}=8$ \\
\hline & hexagonal bipyramidal motif type, $\mathrm{CN}=8$ \\
\hline & $q_{2}$ motif type, $\mathrm{CN}=9$ \\
\hline & $q_{4}$ motif type, $\mathrm{CN}=9$ \\
\hline & $q_{6}$ motif type, $\mathrm{CN}=9$ \\
\hline & $q_{2}$ motif type, $\mathrm{CN}=10$ \\
\hline & $q_{4}$ motif type, $\mathrm{CN}=10$ \\
\hline & $q_{6}$ motif type, $\mathrm{CN}=10$ \\
\hline & $q_{2}$ motif type, $\mathrm{CN}=11$ \\
\hline & $q_{4}$ motif type, $\mathrm{CN}=11$ \\
\hline & $q_{6}$ motif type, $\mathrm{CN}=11$ \\
\hline & cuboctahedral motif type, $\mathrm{CN}=12$ \\
\hline & $q_{2}$ motif type, $\mathrm{CN}=12$ \\
\hline & $q_{4}$ motif type, $\mathrm{CN}=12$ \\
\hline & $q_{6}$ motif type, $\mathrm{CN}=12$ \\
\hline & Voronoi index, number of 3 -edged facets \\
\hline & Voronoi index, number of 4-edged facets \\
\hline & Voronoi index, number of 5-edged facets \\
\hline & Voronoi index, number of 6-edged facets \\
\hline & Voronoi index, number of 7-edged facets \\
\hline & Voronoi index, number of 8-edged facets \\
\hline$\stackrel{2}{2}$ & Voronoi index, number of 9-edged facets \\
\hline$\Xi$ & Voronoi index, number of 10-edged facets \\
\hline ○ & 3-fold symmetry index \\
\hline$>$ & 4-fold symmetry index \\
\hline
\end{tabular}




\begin{tabular}{|l|l|}
\hline 5-fold symmetry index \\
\hline 6-fold symmetry index \\
\hline 7-fold symmetry index \\
\hline 8-fold symmetry index \\
\hline 9-fold symmetry index \\
\hline 10-fold symmetry index \\
\hline Voronoi volume \\
\hline Voronoi area \\
\hline Voronoi volume (mean) \\
\hline Voronoi volume (standard deviation) \\
\hline Voronoi volume (minimum) \\
\hline Voronoi volume (maximum) \\
\hline Voronoi area (mean) \\
\hline Voronoi area (standard deviation) \\
\hline Voronoi area (minimum) \\
\hline Voronoi area (maximum) \\
\hline Voronoi nearest-neighboring distance (mean) \\
\hline Voronoi nearest-neighboring distance (standard deviation) \\
\hline Voronoi nearest-neighboring distance (minimum) \\
\hline Voronoi nearest-neighboring distance (maximum) \\
\hline
\end{tabular}

